\title{
Kernos
}

Revue internationale et pluridisciplinaire de religion grecque antique

19 | 2006

Varia

\section{Micol PERFIGLI, Indigitamenta. Divinità funzionali e Funzionalità divina nella Religione Romana}

\section{Francesca Prescendi}

URL : https://journals.openedition.org/kernos/508

DOI : $10.4000 /$ kernos.508

ISSN : 2034-7871

\section{Éditeur}

Centre international d'étude de la religion grecque antique

\section{Édition imprimée}

Date de publication : 1 janvier 2006

Pagination : 500-502

ISSN : 0776-3824

\section{Référence électronique}

Francesca Prescendi, « Micol PERFIGLI, Indigitamenta. Divinità funzionali e Funzionalità divina nella Religione Romana », Kernos [En ligne], 19 | 2006, mis en ligne le 18 juin 2011, consulté le 24 août 2022. URL : http://journals.openedition.org/kernos/508; DOI : https://doi.org/10.4000/kernos.508 
avec le patronus, puisque «le commanditaire à Rome est le véritable facteur d'un ouvrage d'art». Suit l'article de Christian Jouhaud («'Qu'est-ce un auteur?' de l'Antiquité romaine au XvII ${ }^{e}$ siècle »), qui s'interroge sur la construction de la position d'auteur en mettant en dialogue Rome et le XVII ${ }^{\mathrm{e}}$ s. en Europe, sociétés gouvernées par les liens de patronage et de clientèle, dans une perspective qui privilégie l'interaction entre pratiques d'écriture et cadres sociaux.

La structure interactive ainsi que la qualité des contributions font de ce livre un instrument de réflexion important. Vu la référence omniprésente à Foucault (à l'exception de l'article de Gregory Nagy), les hellénistes ressentiront peut-être le manque d'une mise en évidence plus claire, en guise d'épilégomènes, des divergences latentes par rapport au modèle foucaldien. Peut-être est-ce trop demander à un ouvrage déjà riche et bien orchestré. Débat à poursuivre donc, même si «la mort du sujet » ou « la question homérique » l'ont rendu marginal et aride. Il est temps de se réconcilier avec l'idée que la personne, mythique ou réelle, de l'auteur est un trait particulièrement important du système culturel grec ancien.

Ioanna Papadopoulou

(Université libre de Bruxelles)

Micol Perfigli, Indigitamenta. Divinità funzionali e Funzionalità divina nella Religione Romana, Pisa, Edizioni ETS, 2004. 1 vol. $15,5 \times 22 \mathrm{~cm}, 308$ p. (Antropoi. Studi e materiali di Antropologia storica del mondo antico, 2). ISBN : 88-467-0977-2.

Le livre de Michol Perfigli (M.P.) ouvre à nouveau un des dossiers parmi les plus débattus de la religion romaine, c'est-à-dire celui des divinité fonctionnelles (les Sondergötter), mais il le fait de façon exhaustive et avec des catégories d'analyse jamais utilisées jusqu'ici. La grande valeur de cet ouvrage doit être soulignée dès maintenant. 308 pages de recherches pointues, précises, approfondies, qui nous mènent au centre d'un problème ancien : la classification de leurs divinités par les Romains, mais aussi d'un problème plus récent: les interprétations que les savants modernes en ont données. Il faut le dire clairement, il ne s'agit pas d'un livre aisé. La complexité du sujet et la volonté de M.P. de l'explorer en profondeur ont produit un essai dont la lecture ne permet pas de distractions. Pour se laisser conduire par le grand savoir de l'A., il faut s'engager dans une lecture « religieuse », en prenant ce mot dans l'acception primaire de religio, c'est-à-dire «recueillement et scrupule ».

Le livre se compose de deux parties et trois chapitres différents entre eux par l'ampleur et les enjeux. La première partie (correspondant au premier chapitre), est une dissertation qui occupe plus que la moitié du volume. Ce chapitre, dont le titre «Un dio per ogni cosa » est très parlant, analyse toutes les divinités romaines «mineures », c'est-à-dire celles dont le domaine d'action est assez étroit : par exemple des divinités responsables de quelques gestes faits pendant l'accouchement d'une femme et pendant les rapports sexuels, ou des états d'âme particuliers comme les différents aspects de la peur. À ma connaissance, cette partie du livre est l'unique publication existante qui offre un panorama complet de ces divinités assez méconnues. Il s'agit donc d'un travail nécessaire, même si peut-être un peu moins intéressant au niveau intellectuel que les deux autres chapitres du livre. La matière est très vaste et cela explique quelques inadvertances. On remarque par exemple dans la bibliographie l'absence d'une étude à laquelle on se serait attendu, celle de J.-J. AubERT, «La procréation (divinement) assistée dans l'antiquité gréco-romaine », in V. DASEN (éd.), Naissance et petite enfance dans l'Antiquité. Actes du colloque de Fribourg, 28 nov. - $1^{\prime \prime}$ 
déc. 2001, Fribourg, 2002, p. 187-198. Mais probablement le manuscrit de M.P. était déjà sous presse au moment de la diffusion de ce volume. Contemporain de la parution du livre de M.P. sont aussi les actes Libitina e dintorni. Libitina e i luci sepolcrali. Le leges libitinariae campane. Iura sepulcrorum: vecchie e nuove iscrizioni. Atti dell'XI Rencontre franco-italienne sur l'épigraphie, Rome, 2004, dans lesquels est publié l'article de J. Scheid «Libitina, Lubentina, Venus Libitina et les mort », 13-20, qui aurait obligé M.P. a revoir la partie concernant justement cette divinité ambiguë qu'est Libitina.

À la p. 101, M.P. discute du livre de Philippe Borgeaud, Recherches sur le dieu Pan en présentant l'auteur comme «lo studioso francese ». Ma position actuelle m'oblige de rectifier en précisant qu'il s'agit d'« uno studioso svizzero » et à remarquer la faute de transcription du nom de Borgeaud à la p. 102. Mais ce ne sont là que des coquilles à relever en passant. Dans ce paragraphe M.P. compare des divinités romaines de la peur, Pavor et Pallor, à la divinité grecque de la terreur panique, Pan, et au dieu Phobos. La comparaison amène M.P. a une conclusion intéressante : le fait de condenser la peur dans des figures permet de transformer ce sentiment de négatif en positif. À travers ces divinités, l'homme arrive à s'expliquer le phénomène émotionnel et, surtout pour ce qui concerne la peur sur le champ de bataille, à diriger ce sentiment, en priant le dieu d'intervenir dans le camp des ennemis. Dans cette première partie M.P. se sert de comparaisons avec d'autres cultures pour illustrer le dossier romain, par exemple avec des contes populaires traditionnels de l'Italie et de la Chine. Les considérations que M.P. fait sur la relation entre le genre des divinités et les activités qu'elles patronnent sont celles qu'on pouvait attendre : les déesses, plus nombreuses, interviennent dans les différents moments de l'accouchement, tandis que les dieux s'occupent de la fécondation.

La deuxième partie "Gli indigitamenta » est plus courte et intense. Ici sont débattues à fond les grandes questions que les savants de la religion romaine se sont posées et se posent. Dans le deuxième chapitre «Per una storia degli indigitamenta », M.P. fait le point sur l'histoire des études et met en relief comment les savants modernes ont contribué à créer un casse-tête autour de ce problème. Elle démêle avec patience l'écheveau qui enveloppe deux concepts souvent assimilés, c'est-à-dire celui des indigitamenta et de ces divinités mineures ou Sondergötter. Les indigitamenta sont des listes de divinités, dans lesquelles les dieux sont inscrits à côté de leurs rationes, c'est-à-dire leurs compétences ou domaines d'action. Mais les sources anciennes ne disent pas que ces listes contiennent seulement les noms des divinités «mineures ». Probablement, elles contiennent toutes les divinités. L'identification des indigitamenta et des dieux mineurs remonte au célèbre manuel de Marquardt, Römische Statswervaltung et est reprise par beaucoup d'auteurs postérieurs. M.P. analyse ensuite l'interprétation de Usener, auquel remontent les termes de Augenblickgötter et de Sondergötter, qui ont eu beaucoup de succès. Pour Usener, ainsi que pour d'autres savants du XIX ${ }^{e}$ s., ces divinités remontaient à l'époque la plus archaïque de la religion romaine, l'époque que l'esprit romantique considérait comme primitive et dominée par une peur irrationnelle des dieux. Dans ce type de considérations, remarque M.P., est implicite l'idée d'une évolution de la pensée qui conduit de cette mentalité à celle plus évoluée du monothéisme chrétien. Dans ses conclusions à cette partie, M.P. reprend une idée déjà exprimée auparavant, à savoir que les noms des divinités mineures romaines sont des «images sonores créées pour organiser une série des données de l'expérience humaine relevant d'un point de vue existentiel ». M.P. conclut ensuite qu'une pensée qui ordonne ses connaissances dans un système religieux, n'est pas complètement 
rationnelle, mais pas primitive pour autant, puisqu'elle est capable d'analyser la réalité à travers des instruments d'enquête et des valeurs sociales données.

Le dernier chapitre «Gli antichi e i loro indigitamenta » propose une étude des témoignages anciens sur la signification du mot indigitamenta. Les passages ne sont pas nombreux. Comme c'est souvent le cas dans ce genre d'études antiquaires, le point de départ est représenté par un passage de Servius, le commentateur de Virgile. Ce passage fait comprendre que les indigitamenta sont des listes de divinités : pas seulement les divinités mineures, mais toutes les divinités. Parmi les observations intéressantes de M.P., remarquons la suivante (p. 222) : pour beaucoup de divinités fonctionnelles la ratio équivaut à l'étymologie du nom divin. C'est pour cela que les Romains pouvaient évoquer sans difficultés les noms de leurs dieux en faisant des actions rituelles. La liste des indigitamenta est donc une liste des divinités, qui constitue un support à l'action rituelle et a pour but pratique de permettre à l'officiant d'évoquer la divinité qui convient dans chaque circonstance. Ensuite M.P. analyse la relation entre indigitamenta, incantamenta, carmina, indigitare, imprecari, etc. en mettant en relief que le premier mot comme les autres exprime un pouvoir d'influencer la réalité par la prononciation de paroles. M.P. affirme que les indigitamenta sont un «texte en vers à chanter, dans lequel sont annotées, pour ne pas se tromper, les paroles efficaces à adresser à chaque divinité, dont, avant tout, est enregistré le nom »(p. 240). Sur cette liste, les auteurs antiquaires ont construit leurs étiologies. M.P. se penche ensuite sur le verbe indigitare qui n'indique pas l'action générique de prier une divinité, mais qui est plutôt un terme technique indiquant le fait de l'invoquer avec le nom spécifique lié à une fonction et à un domaine précis. Enfin, M.P. analyse la relation entre indigitare et indiges. L'adjectif indiges pourrait indiquer une divinité qui a été rajoutée dans ces listes des dieux qui sont les indigitamenta. Le fait que ces listes étaient mises à jour par les pontifes est pertinent. M.P. remarque aussi, justement à mon avis, que quand les auteurs anciens rapportent quelques informations sur ces listes, ils le font parce que des innovations ont été apportées.

Les pages 264-265 résument les conclusions de ce travail. Mais un lecteur paresseux qui lirait les conclusions avant d'acheter le livre serait peut-être découragé. En fait, il est impossible de rendre compte, en si peu de place, d'un travail qui plonge dans les mécanismes fondamentaux de la théologie des Romains. Je conseille donc au lecteur de bien vouloir commencer par le début et de suivre attentivement l'ensemble des parcours auquel M.P. nous convie. Parmi ces lecteurs, ceux qu'intéresse la religion grecque trouveront à coup sûr dans cette « réflexion romaine » des points de comparaison extrêmement féconds.

Francesca Prescendi (Université de Genève)

\section{Actes de colloques, ouvrages collectifs et mélanges}

Barringer Judith, Hurwit Jeffrey M. (éds), Periklean Athens and its legacy. Problems and Perspective, Austin, Texas UP, 2005.

J. NeILS, The girl in the pithos: Hesiod's elpis, p. 37-45; H.A. SHAPIrO, The judgement of Helen in Athenian art, p. 47-62; B. SISMONDO RIDGWAY, 'Periklean' cult image and their media, p. 111118; E.B. HARRISON, Athena at Pallene and in the agora of Athens, p. 119-131; J.M. HuRWIT, The Parthenon and the temple of Zeus at Olympia, p. 135-145; J.M. BARRINGER, Alkamenes' Procne and Itys in context, p. 163-176; O. PALAGIA, Interpretations of two Athenian friezes: the temple on the 